\title{
ENSINO DE TÉCNICA DE COMUNICAÇÃO TERAPÊUTICA ENFERMEIRA-PACIENTE - PARTE I*
}

Maguida Costa Stefanelli**

STEFANELLI, M.C. Ensino de técnicas de comunicação terapêutica enfermeira-paciente - Parte I. Rev. Esc. Enf. USP, São Paulo, 20(2):161-183, 1986.

Apresenta-se ampla revisão de literatura sobre comunicação em enfermagem $e$ em enfermagem psiquiátrica. É ressaltada a importância do uso da comunicação terapêtutica pela enfermeira.

E a comunicação que torna possível ao homem existir no mundo em interação com seus semelhantes. Desde o nascimento até a morte e, em todos os momentos da vida, as pessoas vêem-se envolvidas no processo da comunicação, sem perceberem a existência ou a significação da mesma como condição fundamental para o pleno desenvolvimento do ser humano.

O homem vale-se da comunicação em todas as suas experiências de vida, de modo interpessoal ou dual, em pequenos ou grandes grupos; até quando não está em uma dessas situações, se refletir um pouco, perceberá que se encontra sob o impacto ou influência da comunicação. E por meio da comunicação que ele partilha com outras pessoas seus valores, crenças, idéias e sentimentos; a maneira como essas pessoas reagem à comunicação pode gerar satisfação ou insatisfação, ou seja, determinar o sucesso de suas tentativas de ajustamento ao meio em que vive. Pode-se afirmar, então, que a essência do bem-estar do ser humano e da sua saúde mental está diretamente relacionada ou mesmo dependente dos seus padrões de comunicação e de como os outros reagem a eles.

Isto explica o interesse que o estudo da comunicação já despertou e continua despertando em várias áreas do conhecimento, como psicologia,, sociologia, medicina, mais especificamente a psiquiatria e a enfermagem, principalmente a psiquiátrica.

Consoante MAY (1973), entretanto, apesar de vivenciar a era da comunicação, na qual os meios desta se dão de modo cada vez mais rápido, o homem não existe no mundo com os outros; apenas vive no anonimato, com a sensação de vazio e desesperança que o rodeia.

* Extraído da tese de doutoramento apresentada à Escola de Enfermagem da USP.

** Enfermeira. Professor Assistente Doutor do Departamento de Enfermagem Materno-Infantil e Psiquiátrica da Escola de Enfermagem da USP - disciplina Enfermagem Psiquilitrica. 
SULLIVAN (1953a), introdutor da teoria interperssoal no campo da psiquiatria e RUESCH (1957, 1964), estudioso do uso da comunicação terapêutica na relação com o doente mental, declaram ser a doença mental uma perturbação no relacionamento interpessoal e um distúrbio no processo de comunicação da pessoa. Essas afirmações associadas à declaração de MAY (1973), já citada, facilitam a compreensão da importâncià da comunicação na assistência de enfermagem. E enfermeira o membro da equipe terapêutica que mais tempo passa junto ao paciente e que mais oportunidades tem para com ele interagir; seu mister, portanto, é tornar terapêutica a comunicação que desenvolve com 0 paciente ao assistí-lo.

Podemos considerar PEPLAU (1952) e TUDOR (1952) como as introdutoras do uso da comunicação terapêutica em enfermagem, uma vez que esta é o instrumento básico do relacionamento enfermeira-paciente, como preconizado pelas autoras citadas. Estas consideram a enfermagem como relacionamento humano entre a enfermeira e a pessoa necessitada de ajuda.

Em enfermagem psiquátrica a comunicação enfermeira-paciente tem sido mais profundamente estudada, uma vez que a doença mental é, como vimos, considerada por vários autores um problema de comunicação ou de relacionamento interpessoal.

Por considerarmos a comunicação enfermeira-paciente da máxima importância para a eficácia da assistência de enfermagem, julgamos oportuno realizar um estudo mais profundo sobre o uso das técnicas de comunicação terapêutica por alunas de graduação em enfermagem.

Sentimos a necessidade de fazer levantamento extensivo do que as enfermeiras já estudaram sobre comunicação em enfermagem e no relacionamento terapêutico enfermeira-paciente, porque a comunicação terapêutica não foi, ainda, estudada o suficiente para estabelecer um corpo de conhecimentos em enfermagem devidamente comprovados.

O estudo da comunicação interpessoal é muito amplo e tem sido desenvolvido em várias áreas do conhecimento. No presente estudo, entretanto, a revisão de literatura será restrita, dentro do possivel, à área de enfermagem em geral e, mais especificamente, da enfermagem psiquiátrica que é nosso foco de interesse.

Tomamos o cuidado de apresentar a revisão de literatura em seqüência cronológica para que o leitor tenha uma visão do desenvolvimento de estudos sobre a comunicação em enfermagem de seu uso.

\section{Comunicação em enfermagem.}

Em 1859, Florence Nightingale já demonstrava preocupação com a comunicação que se desenvolve entre a enfermeira e o paciente (NIGHTINGALE, 1946). Ao abordar a observação de pacientes, ela faz comentários sobre certos tipos de perguntas vagas ou que provocam respostas imprecisas, a importância da enfermeira sentar-se de frente para 
o paciente e não permitir interrupção da comunicação e recomenda que não se deve dar ao paciente falsas esperanças ou conselhos.

Podemos inferir, portanto, que a comunicação integrava os primeiros instrumentos da assistência de enfermagem tal como a conhecemos hoje.

Segundo PENDALL (1954), a comunicação da enfermeira afeta tanto seu relacionamento pessoal como profissional. Chama a atenção para o fato de que as enfermeiras devem estar atentas para isto, uma vez que elas trabalham com pessoas e não com objetos; afirma o autor que o sucesso de todos os esforços na profissão depende do relacionamento que se desenvolve entre enfermeira e paciente, pessoal da equipe e alunos. Se o relacionamento falhar, a enfermeira falha.

A importância da comunicação para a administração hospitalar é ressaltada por DOANE (1954). Esta afirma que a função mais reconhecida do administrador é estabelecer e manter abertos os canais de comunicação dentro da organização. Para esta autora, considerar as idéias dos outros, manter um clima de respeito mútuo, definir objetivos em termos claros e precisos, ter propriedade tanto nas comunicações orais quanto nas escritas, ajudam a manter comunicação efetiva entre administrador do hospital e pessoal do departamento de enfermagem, o que tem como conseqüência um melhor cuidado de enfermagem.

BECKKER (1955) destaca a importância da competência interpessoal para a estudante de enfermagem e a necessidade de se introduzir o ensino desta nos currículos de enfermagem. Na experiência relatada pela autora, as alunas começam a adquirir esta competência no início do curso, quando interagem entre si e com as docentes em pequenos grupos de discussão; e, mais tarde, continuam no desenvolvimento do curso, quando discutem em sala de aula o relacionamento que ocorre entre enfermeira e paciente na demonstração e execução de cada técnica e em todas as situações de complexidade crescente.

KREUTER (1957), BOJAR (1958) e JACKSON (1959) ressaltam a importância da comunicação para a excelência do cuidado de enfermagem para oferecer segurança ao paciente, compreensão do mesmo e auto-compreensão da enfermeira, com o objetivo de restabelecer no paciente a percepção de que ele é uma pessoa e, também, de facilitar a sua recuperação.

Em toda a obra de ORLANDO (1961), observa-se a importância que a autora atribuiu à comunicação enfermeira-paciente. Ela exemplifica a necessidade de compreensão, pela enfermeira, da comunicação do paciente, por meio de descrições de sua situação de vida; afirma que este, devido à ansiedade e ao medo do que lhe possa acontecer, não consegue se expressar com lógica e clareza, e que a enfermeira precisa explorar a significação da experiência para o paciente, tentando descobrir qual o seu problema real e que só assim é possível identificar a ajuda que ele deseja e da qual necessita. Nas situações que a autora descreve, fica evidente a importância da comunicação adequada entre enfermeira e paciente para o alivio da sensação de dor e mal-estar e para ajudá-lo a enfrentar situaçōes desagradáveis e desconhecidas. Para a autora, a as- 
sistência da enfermagem é fundamentada na interação enfermeira-paque as necessidades do paciente sejam clarificadas e resolvidas em conjunto.

Segundo DAVIS (1963), a importância em desenvolver o relacionamento terapêutico tem sido muito ressaltada por diversos autores; para ela, entretanto, se se estiver realmente interessado em ensinar esta habilidade aos alunos, é necessário fazer muito mais do que simplesmente apresentar-lhe alguns textos. Esta habilidade tem de ser baseada em princípios e conceitos derivados de um corpo de conhecimento específico e de pesquisa, que constituam uma estrutura de referência. A citada autora usa, para o ensino da comunicação enfermeira-paciente, a teoria da comunicação de RUESCH $(1952,1953)$ e utiliza seus princípios, também na comunicação que mantém com os alunos para melhor aprendizado; coloca, como objetivo final do aprendizado, ajudar o aluno a desenvolver sua habilidade em comunicação e, portanto, a ser capaz de manter um relacionamento mais construtivo com seus pares, paciente e familiares.

MEADOW \& GASS (1963) ressaltam a importância da comunicação para a função da enfermeira como entrevistadora, pois esta se vê freqüentemente na situação de entrevistar candidatos a emprego, alunos, ou mesmo pacientes. Para as autoras, há melhora considerável no desempenho desta função quando a enfermeira analisa suas próprias interaçōes, ou seja, tornar-se consciente de suas falhas de comunicação.

WIEDENBACH (1963) diz ser a enfermagem a arte de ajudar o paciente e, para tal, a enfermeira tem de centrar sua atenção na necessidade real de ajudar o paciente; para atender a esta necessidade ela lança mão de todo seu potencial e de todas as suas habilidades, especialmente das referentes à comunicação com pacientes.

MEYERS (1964) relata que, ao ministrar um cuidado de enfermagem, usou três tipos diferentes de comunicação. No primeiro a enfermeira ministrou o cuidado sem nada dizer ao paciente; no segundo, conversou sobre assuntos superficiais, não pertinentes ao cuidado; no terceiro, demonstrou interesse pelo paciente, explicou-lhe o que estava fazendo e o porquê a autora concluiu que o último tipo de comunicação, que chamou de estruturado, foi o mais benéfico para o paciente, pois fê-lo sentir-se tratado como pessoa e provocou diminuição da ansiedade causada pelos problemas inerentes à hospitalização.

Segundo SKIPPER et alii (1964), a comunicação que ocorre entre o paciente hospitalizado e os profissionais que o assistem é extremamente importante. Segundo estes autores, o profissional que interage com 0 paciente de forma clara e objetiva, atendendo a seus interesses e dando-lhe as informações necessárias, é a pessoa que lhe oferece segurança na situação interpessoal e que pode promover satisfação das suas necessidades básicas.

Facilitar a manuntenção de efetiva comunicação, verbal e não verbal, e, promover o relacionamento interpessoal são fatores colocados por 
ABDELLAH \& LEVINE (1965) entre os problemas básicos a serem considerados na assistência de enfermagem.

De acordo com LEWIS (1965), CONANT (1965), DIERS \& LEONARD (1966) e JOHNSON et alii (1967), a comunicação é, quando não o próprio cuidado, parte essencial do mesmo ou do tratamento de enfermagem. Comentam os autores as dificuldades em estudar a comunicação em enfermagem, uma vez que não é fácil a extrapolação de métodos de outras áreas devido à especificidade de cada uma. LEWIS (1965) é enfática quando recomenda a necessidade de se avaliar se a própria comunicação é efetiva para as outras pessoas e salienta a importância da sua correção.

MOSS \& MEYERS (1966), em seu estudo sobre redução ou alívio da dor do paciente, sem uso de drogas, ressaltam a importância da interação enfermeira-paciente afirmando ser este um dos caminhos para aliviar a dor.

ALTSCHUL (1970) comenta a necessidade de encorajar as enfermeiras a falarem com seus pacientes, uma vez que vários estudiosos ingleses e norte-americanos têm apontado a comunicação como uma contribuição importante para o cuidado de enfermagem. Acrescenta que a conversa com o paciente tem um propósito terapêutico, e que, portanto, a enfermeira tem de desenvolver habilidade no uso da comunicação terapêutica.

Segundo KING (1971) e ROGERS (1971), o homem e o seu meio constituem o centro de atenção da enfermagem; portanto, a interação que ocorre entre enfermeira e paciente tem de ser levada em consideração para a eficiência da assistência de enfermagem; é por meio desta interação que a enfermeira identifica os problemas do paciente em relação à experiência vivida no momento, e pode determinar os objetivos de sua assistência.

Para KING (1971), a enfermeira usa a comunicação interpessoal a fim de ajudar o cliente a mover-se em direção à saúde; ela precisa estar atenta à comunicação que ocorre entre ela e o paciente, porque cada experiência é única e não se repete, dependendo de fatores como tempo, local, situação e pessoas envolvidas.

No estudo de SANTOS (1972) ficaram evidentes os problemas de comunicação enfermeira-paciente quando da ministração de medicamentos: a maioria dos pacientes mánifestou o desejo de receber informação sobre a medicação; mais da metade da população não recebeu informação e dos que a receberam somente $2,5 \%$ a consideraram satisfatória; e os pacientes que deixaram de pedir informação o fizeram por receio e desconhecimento de seus direitos. Estes resultados mostram que a comunicação enfermeira-paciente foi inadequada. Segundo a autora, cabe à enfermeira dar o primeiro passo para a interação com o paciente e decidir, de acordo com a situação de cada um de que informação ele necessita $e$ qual lhe pode ser dada.

PEITCHNINS (1972) afirma que, pela revisão da literatura existente, embora as enfermeiras tenham mostrado, no passado, baixos índices 
de competência interpessoal, há evidência de se terem as mesmas tornado conscientes de suas limitações e de se esforçarem por superá-las, desenvolvendo-se neste aspecto.

VENINGA (1973), especialista em comunicação, ao ser internada em um hospital para se submeter a uma cirurgia, pôde vivenciar e avaliar o processo de interação enfermeira-paciente. Afirmou que não há dúvida quanto à importância de comunicação para a vida humana, podendo até significar, vida ou morte para as pessoas, e, que, em nenhum lugar, isto é tão real como quando estão internadas em um hospital.

De acordo com HEIN (1973), a habilidade em comunicação tornou-se a mola mestra para fazer de teorias, conceitos e princípios de enfermagem uma realidade na prática profissional.

JORGE(1974) ressalta a importância do uso da comunicação adequada nas passagens de plantão e afirma que, quando esta não ocorre, há dispersão da atenção do pessoal com prejuízo para o paciente.

WANDELT \& AGER (1974) dão ênfase à comunicação enfermeirapaciente quando colocam, entre os intens importantes para um sistema de avaliação da qualidade de assistência, a ajuda ao paciente na expressão de seus sentimentos, problemas, expectativas e relações interpessoais gratificantes.

PIERINI et alii (1975) destacam a importância do relacionamento enfermeira-paciente, ou seja, o valor da comunicação entre ambos para a satisfação de necessidade básica de segurança do paciente.

KOIZUMI (1975 a, b) ao descrever os fundamentos da assistência de enfermagem deixa evidente a importância da comunicação na valorização da auto-imagem do paciente. Coloca, entre os princípios que fundamentam a assistência, o estabelecimento e manutenção de relacionamento onde haja da parte da enfermeira, acurada percepção do paciente e utilização de todas as formas de interação para atingir a verdadeira individualização de cada ser humano sob sua responsabilidade. O mérito da comunicação enfermeira-paciente é colocado em foco, novamente, pela mesma autora, quando comenta a comunicação da enfermeira com paciente afásico.

HORTA (1975) afirmou ser a comunicação um dos instrumentos básicos para que oprofissional possa desempenhar, cientificamente, suas funções, ao desenvolver os passos do processo de enfermagem; sem a comunicação é impossivel interagir terapeuticamente com o indivíduo e dar-lhe assistência; a dificuldade no uso deste instrumento afeta diretamente a qualidade e quantidade de cuidados que o indivíduo, família e comunidade devem receber. A comunicação é, portanto, para a autora, um elemento indispensável para a assistência de enfermagem.

De acordo com KRIZINOFSKY (1976) a aplicação da teoria da comunicação no campo da enfermagem geral e no da psiquiatria, com evidência na área de saúde mental, tem sido, historicamente, um aspecto integrante do processo de enfermagem; as técnicas utilizadas na comuni- 
cação com pacientes são chamadas de terapêutica, e têm sido preconizadas como base do relacionamento enfermeira-paciente. Afirma a autora que algumas pesquisas têm evidenciado o efeito da aplicação destas técnicas na diminuição da ansiedade, mas sobre a articulação da teoria da comunicação com o processo de enfermagem muito pouco se tem publicado.

COHEN (1976) comenta a impotância da comunicação desde os primórdios da civilização até nossa época, ressaltando a preocupação das escolas em dar formação sobre o assunto; afirma que na enfermagem também há preocupação com a relevância da comunicação; considera a comunicação enfermeira-paciente como um processo dinâmico, uma experiência única que envolve indivíduos: única porque cada um, com suas peculiaridades, age e reage em dado momento, e o que ocorre neste momento nunca é igual ao que ocorreu no passado nem o será em relação a acontecimento futuro. São as pecularidades do individuo e a dimensão de tempo que fazem da comunicação uma experiência única - algo que não pode ser repetido; e afirma que decorrem dai as grandes dificuldades do estudo da comunicação e de se reconhecer como o próprio comportamento afeta o comportamento do outro.

$O$ valor da interação enfermeira-paciente para minimizar a ansiedade das mães em face da hospitalização de seus filhos, é ressaltada por RAMOS \& MORAES (1976).

A importância do processo de comunicação para a supervisão em enfermagem é ressaltada por RODRIGUES (1976).

REZENDE (1976) afirma que a comunicação em enfermagem é um meio para se atingir o indivíduo, a família e a comunidade e um instrumento que ajuda a promover melhor atuação profissional, assim como um dos caminhos para compreender os indivíduos e diminuir a intensidade de seus temores.

NAKAMAE $(1976 a, b)$ inclui no tratamento de enfermagem, para atender à necessidade básica de eliminação, o oferecimento de apoio por meio da comunicação com o paciente (demonstrar aceitação e interesse e ajudá-lo a expressar seus sentimentos negativos, entre outros). Afirma, ainda, que a interação enfermeira-paciente é fundamental no preparo do paciente para alta hospitalar e declara que esta interação favorece o aprendizado e a modificação de seu comportamento.

SOUZA (1976), ao testar o efeito da interação enfermeira-paciente, como método para atender à necessidade de segurança de paciente submetido à cirurgia, conclui que a mesma foi relevante na redução do seu medo e da sua ansiedade. No seu trabalho encontra-se citações de técnicas de comunicaçção terapêutica.

Para WALKE (1977), a sofisticação dos tratamentos tem exigido um número cada vez maior de pessoal especializado para assegurar a efetividade do programa terapêutico do paciente; conseqüentemente, este não conhece as pessoas e não tem com quem falar sobre seus sentimentos. Em geral é a enfermeira que mais tempo passa junto ao paciente e quando 
este percebe que aquela é sensivel aos seus sentimentos, está interessada na sua pessoa e na compreensão de seus problemas e sentimentos, ele sente-se livre para expressá-los.

Segundo EPSTEIN (1977), é preciso que a enfermeira esteja sempre atenta, não só para desenvolver sua habilidade em técnicas de comunicação, como, também, em desenvolver esta habilidade no paciente - ou na pessoa que recebe ajuda - para que possa interagir, tanto com a enfermeira, comunicando suas necessidades como com as outras pessoas que o cercam.

BATTEN (1977) começa seu artigo interrogando-se se a comunicação face-a-face é uma ciência e, também, o que é tão importante nesta comunicação. Comenta que resultados de levantamentos, discussões e estudos com pessoal da própria equipe mostram que a efetividade máxima do administrador depende de sua habilidade de se relacionar face-a-face com outras pessoas, de suas ações e de intuições interpessoais.

GOLÇALVES (1977) ressalta a importância da comunicação enfermeira-paciente no momento de sua admissão ao hospital para sua adaptação à nova situação que experimenta.

FUERST et alii (1977) ressaltam a comunicação como elemento essencial no estabelecimento da relação enfermeira-paciente para que se possam atingir as metas de enfermagem.

COSPER (1977) comenta que se ouve falar, freqüentemente, sobre a necessidade de comunicação com os pacientes e indaga sobre a forma como isto está sendo feito; para ela a comunicação está intimamente relacionada com a manutenção do equilíbrio psicológico e o alívio da ansiedade. Isto é bem evidenciado quando o paciente se encotra com um tipo de cultura novo para ele, a cultura hospitalar, e não compreende o jargão local. Por isso a autora ressalta a necessidade de ser usada uma linguagem que os pacientes entendam.

Para FERRAZ (1978) algo deve ser feito para amenizar a situação de tensão que o paciente tem de enfrentar no centro cirúrgico. Da leitura do seu trabalho depreende-se que isto só pode ser alcançado por meio de comunicação efetiva entre enfermeira e paciente.

A importância da habilidade interpessoal para a enfermagem é resssaltada por YURA \& WALSH (1978) que afirmam, em seu livro, se esperado que os leitores se sintam motivados a rever seus pensamentos e a participar mais plenamente no processo de enfermagem, que exige habilidade intelectuais, interpessoais e técnicas.

DAUBENMIRE et alii (1978) afirmam que, no planejamento do cuidado de enfermagem, a comunicação efetiva, que é importante para todos, assume conotação mais ampla. A ansiedade experimentada pelo paciente o torna mais vulnerável às mensagens verbais e não verbiais do terapeuta. Declaram, no entanto, haver poucas pesquisas sobre a comunicação como um processo e seus efeitos sobre o paciente e a enfermeira, 
na execução do cuidado de enfermagem. Estas afirmações corroboram as de SIMMONS (1969) e PLUCKHAN (1970).

Consoante YEARWOOD-GRAZZETE (1978), a comunicação é uma habilidade aprendida e praticada, na qual todos se desenvolvem à medida que amadurecem. O autor aceita a enfermagem como um processo interativo e compreende que a comunicação é uma habilidade necessária para a aplicação dos princípios de enfermagem. A enfermeira deve, portanto, estar consciente da própria comunicação e de sua significação.

Para VIEIRA (1978) não se pode pensar em efetividade do processo de enfermagem sem comunicação adequada, uma vez que é constante a comunicação do paciente, com pelo menos uma das pessoas que atuam na unidade; ressalta, ainda, a importância da enfermeira estar preparada em técnicas de observação e de entrevista para interagir adequadamente com o paciente e identificar seus problemas e necessidades. Destaca a importância da comunicação no processo ensino-aprendizagem.

Comunicação e aprendizagem para HENDERSON \& NITE (1978) são aspectos básicos de nossa existência. O uso habilidoso da comuni.cação pela enfermeira é indispensável para o reconhecimento dos aspectos emocionais da doença e cuidado de pacientes tanto quanto para promover o relacionamento de ajuda com os mesmos; este relacionamento, por seu turno, afeta todos os resultados dos procedimentos médicos e cirúrgicos e, finalmente, contribui para o bem-estar do paciente. Segundo estas autoras, a enfermeira, para atingir seus objetivos, usa comunicação de alguma espécie com o paciente, seus familiares e os membros da equipe. Comentam que, embora haja a idéia de que a comunicação possa e deva passar a ser usada de modo terapêutico por todos que atuam na área de saúde, os psicoterapeutas e as enfermeiras psiquiátricas já a usam, como tratamento, há algum tempo, com pacientes que sofrem de distúrbios emocionais. Há, entretanto, crescente reconhecimento de que toda a pessoa com doença física tem problemas emocionais, que são mais efetivamente tratados quando há relacionamento construtivo entre enfermeira e paciente, um tipo de relacionamento onde haja mútúa compreensão. As autoras ressaltam ainda, a importância da auto-compre ensão para a compreensão do outro, se a enfermeira estiver realmente dispostas a tornar terapêutica sua comunicação. A enfermeira tem valores, crenças, sentimentos e opiniōes que afetam seu modo de se comunicar com o paciente; ela tem de estar consciente de como é nestes aspectos e avaliar como eles influem na sua comunicação com o outro.

GARCIA (1978) ressalta a importância do processo de comunicação como instrumento fundamental para que a enfermeira obstétrica atinja seus objetivos ao cuidar do binômio mãe-filho.

GRIVER (1979) dá ênfase à habilidade em comunicação para a efetividade da atuação da enfermeira em sala de operações; ressalta a comunicação como elemento básico para o desenvolvimento da capacidade de liderança da enfermeira administradora de contro cirúrgico, que deve ser capaz de comunicar e de persuadir o pessoal, sob sua responsabilidade. do valor dos novos planos e da mudanças dentro da organização. 
Na leitura de KAMIYAMA (1979) fica evidente a importância da comunicação enfermeira-paciente, no atendimento das necessidades básicas do paciente, quando afirma ser necessário que a enfermeira saiba utilizar inteligentemente a comunicação para assegurar a adequada assistência de enfermagem.

AMORIM (1979), em seu trabalho sobre a necessidade de humanizar a enfermagem, deixa evidente a importância da comunicação no relacionamento enfermeira-paciente.

CLARK (1981) diz que as enfermeiras dispendem pouco tempo conversando com pacicntes e, raras vezes, os aspectos psicossociais e emocionais são incluidos nestas conversações; observou, ainda, a presença de comportamentos verbais de dois tipos: um que encoraja a expressão verbal do paciente e outro que bloqueia a comunicação do mesmo. Chegou à conclusão de que a habilidade em comunicação ć essencial tanto para a prática quanto para a educação em enfermagem e, portanto, esta deve ser ensinada.

De acordo com WEBSTER (1981), o ensino da comunicação tem sido negligenciado apesar de ser um aspecto crucial do cuidado de enferma gem ao paciente que está morrendo.

WLODY (1981) afirma ser a comunicação efetiva necessária para o funcionamento de qualquer organização. Declara que o primeiro requisito para coordenar os esforços do pessoal que assiste o paciente é dispor de canais adequados de comunicação e coloca o sucesso da organização na dependência de comunicação adequada. As barreiras a esta devem ser reconhecidas e corrigidas, prontamente, para serem atingidos os objetivos do cuidado do paciente e da instituição, e a satisfação profissional.

EDWARDS \& BRILHART (1981) dão ênfase à comunicação e a colocam como a força motriz de todo cuidado da saúde; declaram que a capacidade da pessoa se comunicar adequadamente com o cliente, em muitas situações, pode ter o significado de vida ou morte.

STEFANELLI (1981) comenta a importância da comunicação enfermeira-paciente, qualquer que seja sua área de atuação; dá ênfase à comunicação terapêutica e ressalta a necessidade de pesquisas sobre o assunto e de mais atenção a este aspecto nos currículos das escolas de enfermagem.

BOWER (1982) afirma que a enfermeira, para avaliar as necessidades do paciente, tem de usar não somente a observação, como também sua habilidade em se comunicar com o mesmo, de tal modo que o paciente a compreenda, ela compreenda o que o paciente diz e que os demais membros da equipe de saúde compreendem o que ambos dizem.

Ao discorrer sobre a natureza e definição da enfermagem BEVIS (1982) afirma que as atividades de enfermagem são desempenhadas com base em três sistemas do paciente: o intrapessoal, o interpessoal e o co 
munitário. Declara, ainda, que, para ajudar o paciente a adptar-se a estes sistemas, a enfermeira usa vários instrumentos e entre eles a comunicação.

SILVA \& CEZARETTI (1982), ao abordarem o atendimento centrado nas necessidades básicas do paciente, ressaltam a importância de se ouvir o mesmo e seus familiares; de se dar oportunidade para que ele expresse seus sentimentos e receios; e, de comunicar-se com ele de modo simples, claro e seguro para esclarecer as suas dúvidas. Estas autoras consideram que a comunicação é o elemento central de todo o relacionamento humano e que toda mensagem deve ser acessível ao receptor. Daí a importância do uso de conceitos e palavras adequadas ao nivel de compreensão de cada um.

SALZANO (1983) da ênfase a importância da comunicação entre o pessoal de enfermagem de centro cirúrgico e o de unidade de internação, para a efetivação da continuidade do cuidado, o que permite a individualização da assistência de enfermagem ao paciente e, portanto, melhor atendimento.

No trabalho de STEFANELLI (1983 b) a comunicação é colocada como fundamental para a vida humana e, conseqüentemente, de suma importância para a enfermagem, uma vez que toda situação de enfermagem envolve seres humanos e é, portanto, uma situação interpessoal. A citada autora chama a atenção para o dever que tem toda enfermeira de tornar terapêutica sua comunicação com o paciente e de desenvolver estudos nesta área para tornar a comunicação um real instrumento básico de enfermagem.

SCHORR (1983) afirma que a situação atual das enfermeiras, pouco valorizada, é atribuída ao fracasso na comunicação das mesmas. Comenta que as enfermeiras realizam um trabalho magnífico, valioso e, ainda assim, são pouco valorizadas e mal pagas. Isto se deve à falha na comunicação da enfermeira com o público, médicos, autoridades governamentais e com os demais membros da equipe de saúde. Valorizando a profissão, automaticamente, ter-se-á melhor assistência de enfermagem.

Ao apresentarem a assistência de enfermagem à mulher no ciclo grávido-puerperal, considerando o aspecto psicossocial do mesmo, BRfTEZ FARIÑA et alii (1984) colocam a comunicação terapêutica como o instrumento que torna possivel o atendimento integral à cliente.

Podemos concluir, pela revisão de literatura internacional e nacionai, que a comunicação não só é um dos instrumentos básicos da assistência de enfermagem em geral, como também a base para que se alcance a excelência na enfermagem, tanto em seus aspectos técnicos e expressivos quanto em seu desenvolvimento como profissão.

Embora na literatura nacional sejam escassos os trabalhos sobre comunicação enfermeira-paciente, percebemos que já há preocupação com o assunto e mesmo esforços de algumas enfermeiras em usá-lo do modo terapêutico. 
A assistência ao doente mental tem sofrido transformação evolutiva, desde seus primórdios até a época atual.

STEFANELLI (1983a), na revisão sobre relacionamento terapêutico enfermeira-paciente, mostra que a enfermeira, devido à evolução técnicocientífico e à humanização da assistência psiquiátrica, teve de repensar suas funçōes tradicionais, puramente técnicas ou mecânicas, que já não eram tão necessárias e mesmo tornaram-se insuficientes em face desses avanços.

A primeira enfermeira que redefiniu as funções da enfermeira psiquiátrica foi a americana PEPLAU (1952, 1959 e 1962). Para esta autora, no desempenho dessas funções a enfermeira estabelece com o paciente uma relação terapêutica muito importante, que tem como instrumento básico a comunicação terapêutica.

Para PEPLAU (1952, 1960, 1962, 1968 a,b, 1970) o básico na assistência ao doente mental é o alívio da ansiedade que ele experimenta. O alívio da ansiedade, pelo menos a graus toleráveis, torna-o capaz de perceber suas reais necessidades, de analisar elementos que lhe são oferecidos pela enfermeira, em situação interpessoal, e de perceber modos mais saudáveis para satisfazer suas necessidades, ou seja, para enfrentar a realidade de modo mais objetivo. É enfática ao afirmar que falar com pacientes torna-se produtivo quando a enfermeira adquire consciência de seus padrões verbais e assume a responsabilidade destes ao conversar com os pacientes. Os princípios desta autora serão abordados com mais profundidade no referencial teórico do presente estudo.

Segundo TUDOR (1952), a atenção da enfermeira que atua na área psiquiátrica deve estar centrada no fato de tornar terapêuticas suas habilidades interpessoais, uma vez que considera a doença mental como distúrbio no processo de comunicação. Ao agir assim, a enfermeira facilita a socialização do paciente, evita seu isolamento e provê meios para que suas necessidades básicas sejam satisfeitas. É por meio da comunicação que a enfermeira consegue identificar as necessidades não satisfeitas do paciente e os problemas daí decorrentes; desde que consiga ajuda-lo a expressar esses problemas, ela se torna apta para obter resultados positivos com a assistencia que lhe presta. A autora relata como desenvolveu o processo interpessoal com paciente doentes mentais considerados crônicos, valendo-se de vários modos terapêuticos de comunicação interpessoal.

A importância da comunicação que ocorre entre enfermeira e paciente preocupava também os psiquiatras na mesma época em que Peplau e Tudor lançavam as bases do relacionamento enfermeira-paciente.

CAUDILL et alii (1952), em estudo realizado, em 1951, sobre estrutura social e processo de interação em unidade psiquiátrica, criticam a enfermeira que aborda o paciente com perguntas estereotipadas e que faz sugestões vagas, sem a preocupação de validação. 
Na década de 50, após as trabalhos pioneiros de PEPLAU (1952) e TUDOR (1952), seguiram-se vários outros estudos do assunto e, até hoje percebe-se, pela literatura de enfermagem, a preocupação das enfermeiras em tornar produtiva e terapêutica a comunicação que ocorre entre elas e os paciente que assistem.

Enfermeiras, atuando na área de enfermagem psiquiátrica, como KANDLER et alii (1952), BEHYMER (1953, 1957), BERNSTEIN et alii (1954), MORIMOTO (1955), GILBERTSON (1957), entre outros, demonstram preocupação com a comunicação no relacionamento enfermeira-paciente, com o ensino desta, seus efeitos sobre o paciente e a enfermeira e reforçam a necessidade de métodos para avaliá-la.

BEHYMER (1953), em seu estudo, percebeu que seis estudantes de enfermagem, observadas sistematicamente, gastavam menos de um terço de seu tempo interagindo com os pacientes e que o restante era gasto com o pessoal da unidade. Segundo BEHYMER (1957), pouca atenção tem sido dada ao aprendizado da habilidade em comunicação interpessoal. Para comunicar-se com o paciente, a enfermeira necessita tornar-se consciente do que ela comunica e como esta comunicação o afeta; ela deve comunicar ao outro que ele existe como pessoa e que sua participação é esperada.

Para GREGG (1954 e 1963), a enfermeira tem de possuir habilidade interpessoal para ajudar o paciente quando este é assistido na unidade de internação. Como participante do processo de internação com o paciente, ela tem de estudar não só a comunicação dele como também a sua própria e aprender a usá-la em sua experiência interpessoal, tornado-a terapêutica.

HOLMES(1960) chama a atenção para a comunicação que ocorre entre o professor e o aluno: o professor, muitas vezes, é surpreendido usando chavões que podem ser interpretados erroneamente pelos alunos, e que poderão afetar a comunicação destes com seus paciente; os alunos temerosos de não serem suficientemente terapêuticos, ou de se envolvorem com os pacientes, não conseguem se comunicar de modo efetivo.

ROBINSON (1960) e ARTEBERRY (1965) comentam os problemas, as dificuldades e a satisfação que encontraram no relacionamento com pacientes esquizofrênicos. As autoras afirmam ser a comunicação o principal problema da esquizofrenia e que a enfermeira deve se esforçar por resolvê-los porque esta barreira pode ser vencida, apesar de seu um desafio.

MATHENEY \& TOPALIS (1962), MERENESS \& KARNOSH (1964), STRONG (1970), entre outros, declaram que o sucesso do cuidado prestado aos doentes mentais depende da habilidade da enfermeira em comunicação interpessoal; para tanto, não precisa adquirir conhecimentos sobre princípios de comunicação efetiva.

Dos elementos da equipe que assistem o paciente em unidade psiquiátrica, a enfermeira, segundo MELLOW (1966) e TRAIL (1966), encontra-se em situação privilegiada, pois, pode manter-se em interação com o paciente por tempo mais prolongado. $\mathrm{E}$ responsabilidade dela, portanto, 
desenvolver com c paciente experiência interpessoal saudável, para que este adquira padrões de comportamento mais aceitos socialmente. Assim, ela o estará ajudando a recuperar sua saúde mental.

Para JOHNSON \& MILLER (1967), se a enfermeira responder automaticamente à comunicação do paciente, ela reforçará a visão negativa que ele tem de si mesmo e de seus velhos padrões de relacionamento com os outros; neste caso, o paciente nada aprende de construtivo na sua relação com a enfermeira. Nesta interação ele deveria aprender alguma coisa nova sobre si mesmo ou sobre como as pessoas o consideram. A enfermeira precisa, portanto, manter-se atenta a seus modos de comunicação para que esta tenha efeito benéfico sobre o paciente.

TOPF (1969), enfermeira preocupada com o ensino de comunicação terapêutica, ressalta a importância desta para a assistência de enfermagem. Afirma que a tendência no aprendizado da comunicação enfermeira-paciente é a aquisição, por parte da enfermeira, de habilidade em tornar-se terapêutica na interação com o paciente $e$ ir abandonando, gradativamente, os modos não terapêuticos.

A importância dada à comunicação por TRAVELBEE (1969) fica evidente quando ela afirma que esta foi um dos primeiro métodos usados pela enfermeira para atingir sua meta - ajudar o paciente a compreender seus problemas e enfrentá-los. Declara que a comunicação é o meio para a pessoa aprender a se conhecer, embora reconheça que seja árdua a tarefa de se receber uma mensagem, analisá-la criticamente, aprender com ela e agradecer por existir um ser humano que se preocupa o suficiente para compartilhar as suas próprias verdades.

Afirma que é por meio da interação ocorrida entre enfermeira e paciente que este pode mudar seus padrões de comportamento, quando a comunicação é usada adequadamente com fins construtivos.

HAYS \& LARSON (1970), em seu livro, procuraram sistematizar o ensino de comunicação enfermeira-paciente e apresentam uma série de técnicas de comunicação terapêutica e de modos não terapêuticos de comunicação. Analisando-o no seu todo, podemos dizer que este contém os procedimentos básicos da assistência ao doente mental, isto é, como relacionar-se com ele. As autoras não apresentam as técnicas de comunicação terapêutica como resposta às dúvidas da enfermeira ao interagir com o paciente, mas como princípios orientadores da interação. O livro parece um esforço das autoras em colaborar para sanar uma falha dos currículos de enfermagem, ou, como elas próprias dizem, o escasso preparo para a importante função de falar com pacientes. Quando a interação verbal é de natureza terapêutica, construtiva, ela se torna o instrumento fundamental para satisfação das necessidades emocionais do paciente; a pessoa nunca está apenas fisicamente doente, ela sente ansiedade, medo, tristeza e raiva em face da doença e do tratamento, e assim, cada enfermeira, independentemente do local em que trabalha, tem a responsabilidade de tornar o cuidado integral uma realidade. O papel da enfermeira, para estas autoras, é proporcionar ao paciente a aportunidade de identificar e explorar seus problemas no relacionamento com outras pessoas; 
tentar descobrir modos saudáveis de satisfazer suas necessidades emocionais e experimentar um relacionamento interpessoal sadio. Para tanto, a enfermeira tem de tornar o seu papel explícito para o paciente, pois este está acostumado a receber cuidados apenas para atender aos problemas da área física. Comentam, ainda, que poucas enfermeiras têm recebido preparo adequado para desempenhar este papel.

O objetivo de HAYS (1966) e HAYS \& LARSON (1970) não é a proposição de um modelo de entrevista psiquiátrica, mas de princípios que possam ser úteis para tornar terapêutica a comunicação que a enfermeira tem com o paciente no seu dia a dia. Não consideram esgotadas as técnicas, nem como um fim em si mesmas; afirmam que elas têm um papel restrito, mas ajudam a limitar o uso de padrões não terapêuticos de comunicação e tornam a enfermeira mais consciente de sua atuação quando intrage com o paciente.

Consoante GERBER \& SNYDER (1970), a compreensão da linguagem do paciente é o primeiro passo que o terapeuta dá para respeitá-lo como ser humano peculiar; se isto não for considerado, dificilmente penetraremos no seu mundo.

Segundo JOHNSTON (1971), a enfermeira eficiente deve explorar seus próprios sentimentos e comportamentos; só após compreendê-los é que se torna capaz de compreender a motivação do paciente do ponto de vista dele e que isto só pode ser feito quando a enfermeira adquire habilidade em comunicação.

Segundo FIELD (1972), a enfermeira tem a responsabilidade de ajudar e orientar os pais a analisarem os padrões de comunicação que usam no relacionamento com seus filhos, pois o desenvolvimento da doença mental pode ser influenciado pelo tipo de comunicação estabelecido na infância.

MINZONI et alii (197) consideram a enfermagem em saúde mental e psiquiátrica como um processo interpessoal para ajudar o indivíduo a promover saúde mental, prevenir doença mental e auxiliar no tratamento e reabilitação daqueles que enfrentam a doença. Esta citação coincide com as afirmações de TRAVELBEE (1969) e de MANFREDA (1973).

AGUIIERA (1977) e SMTTH (1979) abordam a importância da enfermeira ajudar o paciente a se sentir valorizado como pessoa e de lhe oferecer oportunidades para se comunicar com outras pessoas que queiram ajudá-lo a fim de que ele possa desenvolver um tipo de relacionamento funcional dentro da sociedade. Afirmam que a habilidade em comunicar-se terapeuticamente pode ser aprendida.

Segundo TOPALIS \& AGUILERA (1978), no relacionamento com o paciente, cada enfermeira tem uma contribuição peculiar a oferecer, como pessoa que é, diferente de qualquer outra. Esta é uma das razões por que, em enfermagem psiquiátrica, não há padrões de respostas à perguntas do paciente; há princípios, direções a serem seguidos. A mesma palavra, dita por diferentes enfermeiras, pode ter significados diferentes para o mesmo paciente. Ela deve saber quando falar e quando permanecer em silêncio. 
ARANTES et alii (1979), STEFANELLI (1981) e STEFANELLI et alii $(1981,1982)$ deixam evidente, em seus trabalhos, a importância da comunicação enfermeira-paciente para o desenvolvimento da assistência ao paciente por meio do relacionamento terapêutico. Várias técnicas terapêuticas de comunicação podem ser percebidas nos relatos de experiência e exemplos dados, pela autoras, embora não tenham sido destacadas como tal.

Segundo KALKMAN (1980), facilitar a comunicação é a primeira tarefa da enfermeira no relacionamento terapêutico. Considera a autora ser a dificuldade na comunicação um distúrbio comum aos pacientes com manifestações de doença mental e que o terapeuta deve ter habilidade para ajudar o paciente a se expressar livremente.

WRIGTH \& BURGESS (1981) valorizam a habilidade na comunicação verbal entre enfermeira e paciente e afirmam ser este o aspecto fundamental da assistência; segundo estas autoras, é por meio destas expressões verbais que os problemas são identificados, o relacionamento é desenvolvido, os objetivos são definidos e a intervenção de enfermagem é efetuada.

Para KARSHMER (1982), tratar de doentes mentais e trabalhar com eles não é tarefa fácil para as enfermeiras, mesmo para as mais especializadas. Segundo a autora, embora os currículos de enfermagem incluam o ensino de relacionamento terapêutico, as alunas, em geral, têm medo de interagir com paciente com distúrbios psiquiátricos, talvez devido à idéia preconcebida de que a menor falha ser-lhe-á prejudicial. Ressalta, além disso, que a literatura em geral apresenta apenas receitas de abordagem tão imprecisas que a aluna aprende, apenas, a usar frases estereotipadas para tentar reconfortar o paciente, sem obviamente, consegui-lo. Em geral, as técnicas são apresentadas sem a avaliação de sua significação, de sua propriedade ou do objetivo de seu emprego. A citada autora, apresenta, em seu artigo, oito regras reconhecidas como eficientes, surgidas do trabalho com estudantes do curso de graduação em enfermagem; chama a atenção para o fato de que estas regras não devem ser tomadas como evangelho, mas como sugestões para guiar a comunicação da enfermeira com o paciente e possibilitar tratamento mais humano para o mesmo. As sugestões apresentadas pela autora são: 1) existe uma hierarquia de questões e respostas, que deve ser lembrada - a descrição da experiência, os pensamentos e sentimentos sobre a mesma; 2) deve-se sempre explorar a significação que a experiência tem para o paciente; 3) nunca pressupor coisa alguma; 4) tornar explícito o implícito; 5) eivtar perguntas que levem a respostas monossilábicas; 6) quando o paciente falar sobre pessoas e coisas, centrar a atenção no seu relacionamento com as pessoas; 7) sempre estar atenta às particularidades de tempo, lugar e situação; 8) falar somente por si própria, a aluna sabe o que pensa e sente mas, o que o paciente pensa e sente, ela só pode inferir pelo comportamento dele.

Afirma KARSHMER (1982), ainda, que a aluna deve ser encorajada a analisar criticamente as razões e bases conceituais de cada encontro que tem com o paciente; esta medida estimula a auto-exploração levando-? 
a pensar ativamente sobre o que diz, pensa e sente, onde quer chegar, e que objetivos o paciente e ela têm para cada interação.

HABER (1982) define comunicação como um processo interativo, por meio do qual a pessoa desenvolve sua personalidade e sua habilidade no relacionamento com outras pessoas. Para a autora, enfermeira e cliente estão continuamente em comunicação interpessoal. $\mathrm{O}$ objetivo da enfermeira é adquirir habilidade em comunicação, o que facilita o início, o desenvolvimento e a manutenção do relacionamento com clientes e colegas. Para o cliente, facilita a auto-exploração, auto-compreensão e mudança de comportamento. Esta habilidade ajuda tanto a enfermeira quanto o paciente a desenvolverem relações interpessoais efetivas.

A preocupação quanto à utilização da interação interpessoal em enfermagem psiquiátrica, pode ser percebida em algumas dissertações de mestrado sobre o assunto (GATTÁS, 1981; LEMOS, 1982 e GOULART, 1982.

IVESON-IVESON (1983) faz considerações sobre como a enfermeira interage com seus clientes e colegas e sobre a habilidade necessária para tornar esta interação efetiva; ressalta a importância da enfermeira conhecer os princípios de comunicação a fim de criar um tipo de relacionamento com o paciente que o ajude na sua recuperação. Comunicação é interação entre indivíduos e não apenas manutenção de conversa.

STEFANELLI (1983a), ao explicar o porquê da introdução do relacionamento terapêutico no ensino e prática da enfermagem psiquiátrica, ressalta a importância da comunicação na assistência de enfermagem, considerando que os pacientes desta área em geral viram-se, por algum motivo, impedidos de interagir ou de comunicar-se satisfatoriamente com pessoas de seu meio.

Da revisão feita sobre comunicação em enfermagem psiquiátrica, fica evidente a importância que esta adquire, não só para a saúde mental como, também, quando se analisa a doença mental sob o prisma de disturbio na comunicação; e, a ênfase dada ao uso consciente da comunicação para o atendimento das necessidades do ser humano, como um todo, em constante interação com seus semelhantes. Os autores são enfáticos ao destacarem a necessidade da enfermeira aproveitar todos seus momentos de comunicação com o paciente para torná-los terapêuticos. É imperioso, portanto, que ela adquira habilidade do uso da comunicação efetiva no relacionamento com o paciente, que faça pesquisa sobre o assunto e que divulgue seus resultados. Acreditamos que a área expressiva da enfermagem poderá, assim, ser desenvolvida em sua plenitude.

STEFANELLI, M.C. Teaching communication therapeutic techniques in nurse-patient relationship - Part I. Rev. Esc. Enf. USP, São Paulo, 20(2):161-183, 1968.

In this paper the author presents a literature review about communication in nursing and psychiatric nursing. She emphasyses the use of therapeutic communication by nurse. 


\section{REFERENCIAS BIBLIOGRÁFICAS}

ABDELLAH, F.G. \& LEVINE, E. Conceptualization in nursing research. In: Better care through nursing research. Macmillan, New York. 1965. cap. 4, p.68-90.

AGUILERA, D.C. Components of psychiatric nursing. In: Roview of psychiatric nursing. Saint Louis, C.V. Mosby, 1977. cap. 3, p.37-58.

ALTSCHUL, A.T. Go and talk to the patients. Nurs. Mirror, Sussex, 130(15):41-6, Apr. 10, 1970.

AMORIM, M.J.A.B. Enfermagem - profissão humanitária? Rev. Bras. Enf., Brasília, 32(4): 359-68, out./dez. 1979.

ARANTES, E.C. et alii. Relacionamento terapêutico: considerações teóricas e relato de uma experiência. Rov. Esc. Enf. USP., São Paulo, 13(3):217-23, dez. 1979.

ARTEBERRY, J.K. The disturbed communication of a schizophrenic patient. Perspect. Psychiatr. Care, Hillsdale, 3(5):25-37, Sept./Oct. 1965.

BATteN, J.D. Face to face communication. Nurs. Digest, Wakefield, 5(1):89-90, Feb. 1977.

BECKER, R.S. Interpersonal relations in the basic program. Amer. J. Nurs., New York, 55(8) :952-5, Aug. 1955.

BEHYMER, A.F. Interaction patterns and attitudes of affillate students in a psychiatric hospital. Nurs. Outlook, New York, 1(4):205-7, Apr. 1953.

Patient care: meeting the needs of emotionally ill patients through nursing. In: NATIONAL LEAGUE FOR NURSING. Concepts of nursing care. New York, 1957. Section A, p.1-6.

BERNSTEIN, L. et alii. Teaching nurse-patient relationships: an experimental study. Nurs. Res., New York, 3(2):80-4, Oct. 1954.

BOJAR, S. The psychotherapeutic function of the general hospital nurse. Nurs. Outlook, New York, 6(3):151-3, Mar. 1958.

BRfTEZ FARINA, E. et alii. Assistência de enfermagem à mulher no ciclo grávido-puerperal: aspecto psicossocial. Rev. Esc. Enf. USP, São Paulo, 18(1):5-12, abr. 1984.

BOWER, F.L. A model for implementing the planned care: the nursing care plan. In: The process of planning nursing care. 3. ed. Saint Louis, C.V. Mosby, 1982. cap. 6, p.169-95.

CAUDILL, W. et alii. Social structure and interaction process on a psychiatric ward. Amer. J. Orthopsychiatr., New York, 22(2):314-34, Mar. 1952.

CLARK, J.M. Communication in nursing. Nurs. Times, London, $77(1): 12-8$, Jan. 1, 1981.

COHEN, S.P. Communication. In: SUNDEEN, S.J, et alii. Nurse client interaction: implementing the nursing process. Saint Louis, C.V. Mosby, 1976 . cap. 4, p.68-95.

CONANT, L.H. Use of Bale's interaction process analysis to study nurse-patient interaction. Nurs. Res., New York, 14(4):304-9, Dec. 1965.

COSPER, B. How well do patients understand hospital jargon? Amer. J. Nurs., New York, 77(12) :1932-4, Dec. 1977.

DAUBENMIRE, M.J. et alli. A methodologic framework to study nurse-patient communication. Nurs. Res., New York, 27(5):303-10, Sept./Oct. 1978.

DAVIS, A.J. The skill of communication. Amer. J. Nurs., New York, 68(1):66-70, Jan. 1963.

DIERS, D. \& LEONARD, R.C. Interaction analysis in nursing research. Nurs. Res., New York, 15(3):225-8, Sept. 1966. 
DOANE, E.H. Making communications effective. Nurs. Outlook, New York, 2(8):420-2, Aug. 1954.

EDWARDS, B.J. \& BRILHART, J.K. Communication in nursing practice. Saint Louis, C.V. Mosby, 1981. 245 p.

EPSTEIN, C. A estudante de enfermagem. In: Interasão efetiva na enfermagem. São Paulo, E.P.U., 1977. cap. 9, p. 147.

FERRAZ, E.R. Focalizando o paciente no centro cirúrgico. Hev. Esc. Enf. USP, São Paulo, 12(3):167-9, dez. 1978 .

FIELD, Jr., W.E. Watch your message. Amer. J. Nurs., New York, 72(7):1278-80, July 1972.

FUERST, E. V. et alii. Uso das habilidades comunicativas. In: Fundamentos de enfermagem. Rio de Janeiro, Interamericana, 1977. cap. 13, p. 113-22.

GARCLA, T.J.M. Aplicação do processo de comunicação pela enfermeira obstétrica. Enf. Novas Dimens., São Paulo, 4(6):303-7, nov./dez. 1978.

GATTAS, M.L.B. O relacionamento de pessoa a pessoa: vivência da enfermeira com pacientes psiquiátricos em unidade de hospital geral. Ribeirão Preto, 1981. 215p. (Dissertação de Mestrado - Escola de Enfermagem de Ribeirão Preto da USP).

GERBER, C.B. \& SNYDER, D.F. Language and thought. Perspect. Psychiatr. Care, Hillsdale, 8(5) :230-7, Sept./Oct. 1970 .

GILBERTSON, E. The challenge of communication in interpersonal relations. In: NATIONAL LEAGUE FOR NURSING. Concepts of nursing care., New York, 1957. Section A, p. 16-7.

GONÇALVES, M.M. da C. Estudo de respostas emocionais apresentadas pelo paciente por ocasião da internação hospitalar. São Paulo, 1977. 84p. (Dissertação de Mestrado Escola de Enfermagem da USP).

GOULART, M.C.S. O enfermeiro como elemento de reabilitação do doente mental crônico hospitalizado. Ribeirão Preto, 1982. 127p. (Dissertação de Mestrado - Escola de Enfermagem de Ribeirão Freto da USP).

GREGG, D.E. The psychiatric nurse's role. Amer. J. Nurs., New York, 54(7):848-51, July 1954. The therapeutic roles of the nurse. Perspect. Psychiatr. Care, Hillsdale, 1(1) :18-24, Jan./Feb. 1963.

GRIVER, J.A. Communication skills for getting ahead. AORN J., Denver, 80(2):242-9, Aug. 1979.

HABER, J. Facilitative communication. In: HABER, J. et alii. Comprehensive psychiatric nursing. 2. ed. New York, MacGraw-Hill, 1982. cap. 11, p.231-47.

HAYS, J.S. Analysis of the nurse-patient communications. Nurs. Outlook, New York, 14(9): 32-5, Sept. 1966.

HAYS, J.S. \& LARSON, K. Interacting with patients. New York, Macmillan, 1970, 282p.

HERN, E. Communication in nursing practice. Boston, Little Brown, 1973. 242p.

HENDERSON, V. \& NITE, G. Communications, human relations, learning, health goals and guidance. In: — Principles and practice of nursing. 6. ed. New York, Macmillan, 1978. Cap. 16, p.911-91.

HOLMES, M.J. What's wrong with getting involved? Nurs. Outlook, New YorC, 8(5):250-1, May 1960. 
HORTA, W. de A. Avaliaçăo do histórico de enfermagem por estudantes da Escola de Enfermagem da Universidade de São Paulo, Enf. Novas Dimens., Săo Paulo, 1(4):198-202, set./out. 1975.

Processo de enfermagem. São Paulo, E.P.U., 1979. 99p.

IVESON-IVESON, J. The art of communication. Nurs. Mirror, Sussex, 156(5):47-8, Feb. 2, 1983.

JACKSON, J.K. Communication is important. Amer. J. Nurs., New York, 69(1):90-3, Jan. 1959.

JOHNSON, B.S. \& MILLER, L.C. The interpersonal reflex psychiatric nursing. Nurs. Outlook, New York, 15(5):60-3, May 1967.

JOHNSON, J.E. et alii. Interpersonal relations: the essence of nursing care. Nurs. Forum, Hillsdale, 6(3):324-34, Aug. 1967.

JOHSNTON, M.K. Communication. In: — Mental health \& mental illness. Philadelphia, J. B. Lippincott, 1971. cap. 5, p.19-25.

JORGE, D.R. Efetividade da comunicacão do pessoal de enfermagem na passagem do plantão. Lev. Bras. Ent., Rio de Janeiro, $27(2): 150-68$, abr./jun. 1974.

KALKMAN, M. Individual psychotherapy. In: KALKMAN, M. \& DAVIS, A. J. New dimensions in mental health psychiatric nursing. New York, McGraw-Hill, 1980. cap. 22, p.478-98.

KAMIYAMA, Y. Assistência centrada na identidade social: aspectos psicossociais do cuidado de enfermagem ao paciente de hepatite infecciosa. Săo Paulo, 1979. 191p. (Tese de Livre Docência - Escola de Enfermagem da USP).

KANDLER, H. et alii. A study of nurse-patient interaction in a mental hospital. Amer. J. Nurs., New York, 52(9):1100-3, Sept. 1952.

KARSHMER, J.F. Rules of thumb: hints for the psychiatric nursing student. J. Psychosoc. Nurs. Ment. Health Serv. Thorofare, 20(3) :25-31, Mar. 1982.

KING, I.M. Dynamics of nursing. In: York, John Wiley, 1971. cap. 6, p.87-106.

KOIZUMI, M.S. O atendimento da enfermeira em relação à necessidade de auto-imagem. Enf. Novas Dimens., São Paulo, 1(2):69-74, maio/jun. 1975. 203-8, set./out. 1975(b).

KREUTER, F.R. What is good nursing care? Nurs. Outlook, New York, 5(5):302-304, May 1957.

KRIZINOFSKY, M.T. Evolution of the communication model of therapy. In: KNEISL, C.R. \& WILSON, H.S. Current perspectives in psychiatric nursing. Saint Louis, C.V. Mosby, 1976. cap. 14, p.148-68.

LEMOS, V. de B.S. Reflexões em torno do relacionamento enfermeiro psiquiatrico-paciente, baseado na história da pessoa. Ribelrão Preto, 1982. 211p. (Dissertação de Mestrado Escola de Enfermagem de Ribeirão Preto da USP).

LEWIS, G.K. Communication: a factor in meeting emotional crisis. Nurs. Outlook, New York, 13(8):36-9, Aug. 1965.

MATHENEY, R.V. \& TOPALIS, M. Principios generales de enfermeria psiquiátrica: la enfermera $y$ el paciente. In: Enfermeria psiquíatrica. 3. ed. México, Interamericana, 1962 . cap. 6, p.76-97.

MAY, R. O homem a procura de il mesmo. Petrópolis, Vozes, $1973.230 p$. 
MEADOW, L. \& GASS, G. Problems of the novice interviewer. Amer. J. Nurs., New York, 63(2):97-9, Feb. 1963.

MELLOW, J. Nursing therapy as a treatment and clinical investigative approach to emotional illness. Nurs. Forum, Hillsdale, 5(3):64-73, Aug. 1966.

MERENESS, D.A. \& KARNOSH, L.J. Técnicas de comunicación en la enfermería psiquiátrica. In: - Elementos de enfermería psiquiátrica. México, La Prensa Médica Mexicana, 1964 . cap. 4, p.32-44.

MEYERS, M.E. The effects of types of communication on patient reactions to stress. Nurs. Res., New York, 13(2):126-31, Aug. 1964.

MINZONI, M.A. et alii. Enfermagem em saúde mental. Enf. Novas Dimens., Săo Paulo, 3(5) :350-5, nov./dez. 1977 .

MORIMOTO, F.R. A technique for measuring interactions of patients and personnel in mental hospitals. Nurs. Res., New York, 4(2):74-8, Oct. 1955.

MOSS, F.T. \& MEYERS, B. The effects of nursing interaction upon pain relief in patients. Nurs. Res., New York, 15(4):303-6, Dec. 1966.

NAKAMAE, D.D. Eliminação: uma necessidade básica do homem. Rev. Bras. Enf., Brasillia, 29(1):80-7, jan./mar. 1976(a).

NAKAMAE, D.D. Preparo do paciente para a alta hospitalar: descrição de uma situação. Bov. Bras. Enf., Brasília, 29(2):36-9, 1976(b).

NIGHTINGALE, F. Notes on nursing. 2. ed. Philadelphia, Lippincott, 1946. p.54-76.

ORLANDO, I.J. The dynamic nurse - patient, relationship: function, process and principles. 16. ed. New York, G.P. Putnam's, 1961. 91p.

PEITCHNINS, J.A. Therapeutic effectiveness of counseling by nursing personnel: review of the literature. Nurs. Res., New York, 21(2):138-48, Mar./Apr. 1972.

PHNDALL, R.J. Speaking with people. Nurs. Outlook, New York, 2(2):96-7, Feb. 1954.

PEPLAU, H.E. Interpersonal relations in nursing. New York, G.P. Putnam's, $1952.330 \mathrm{p}$.

Principles of psychiatric nursing. In: ARIETI, S. American handbook of psychiatry. New York, Basic Books, 1959 . cap. 92, p.1840-56.

Talking with patients. Amer. J. Nurs., New York, 60(7):964-6, July 1960.

Interpersonal techniques: the crux of psychiatric nursing. Amer. J. Nurs., New York, 62(6):50-4, June 1962.

264-70, Nov./Dec. 1968(a).

1968. 60p. (b).

Principios básicos para orientación del paciente. Washington, OPAS/OMS,

Foreword. In: HAYS, J.S. \& LARSON, $K$. Interacting with patients. New York, MacMillan, 1970 . p. vil-x.

PIERINI, M.A. et alii. Atuação da enfermeira em exames e cirurgias endoscópicas urológicas. Finf. Novas Dimens., Såo Paulo, 1(5):276-85, nov./dez. 1975.

RAMOS, T.A.G. \& MORAES, E. Papel da enfermeira junto a mães de crianças hospitalizadas. Rev. Bras. Enf., Brasilia, 29(2):45-55, abr./jun. 1976.

REZENDE, A.L.M. de. O processo de comunicação como instrumental da enfermagem. Enf. Novas Dimens. São Paulo, 2(2):98-111, maio/jun. 1976. 
ROBINSON, A.M. Communicating with schizophrenic patients. Amer. J. Nurs., New York, $60(8): 1120-3$, Aug. 1960.

RODRIGUES, M.L. A comunicação no processo de supervisão em enfermagem. Rev. Bras. Ent. Brasilia, 29(2):91-6, abr./jun. 1976.

ROGERS, M.E. Man: a unified whole. In: - An introduction to the theoretical basis of nursing. Philadelphia, F.A. Davis, 1971. cap. 6, p.43-8.

RUESCH, J. Disturbed communication. New York, Norton, 1957. 337p.

The therapeutic process from the point of view of communication theory. Amer. J. Orthopsychiat., New York, 22(4):690-700, July 1952.

$16(3): 215-43,1953$

Synopsis of the theory of human communication. Psychiatry, Washington,

Comunicación terapéutica. Buenos Aires, Paidos, 1964.

SALZANO, S.D.T. Instrumento de comunicacão e enfermagem: estudo da implantaçao de um modelo de comunicacão escrita entre as equipes das unidades cirúrgicas e do centro cirúrgico. Rov. Esc. Enf. USP, São Paulo, 17(3):235-52, dez. 1983.

SANTOS, A.L.V. dos. Contribuição ao estudo da comunicação paciente-equipe de enfermagem, relativa à medicação. São Paulo, 1972. 49p. (Tese de Doutoramento - Fscola de Finfermagem da USP).

SCHORR, N.A. Communicating for success. Int. Nurs. Rev., Geneva, 30(3):73-6, May/June 1983.

SILVA, M.D.A.A. da \& CEZARETTI, I.V.R. Assistencia de enfermagem ao paciente cirúrgico centrada nas necessidades básicas. Rev. Paul. Ent., Såo Paulo, 2(2):35-41, nov./dez. 1982.

SKIPPER, J.K. et alii. What communication means to patients. Amer. J. Nurs., New York, 64(4) :101-3, Apr. 1964.

SMITH, C. Communication skills. Nurs. Times, London, 75(22):926-29, May 31, 1979.

SOUZA, M.F. Efeito da interação enfermeira-paciente como método de atendimento à necessidade de segurança do paciente cirúrgico. Porto Alegre, 1976. 54p. (Tese de Livre Docência - Escola de Enfermagem da UFRS).

STEFANELLI, M.C. Importância do processo de comunicação na assistência de enfermagem. Rev. Esc. Enf. USP., São Paulo, 15(3):239-45, đez. 1981.

Relacionamento terapêtico enfermeira-paciente. Hev. Esc. Enf. USP., São Paulo, 17(1):39-45, abr. 1983 (a).

jun. 1983 (b).

A comunicação terapeutica. Rev. Paul. Enf., São Paulo, 3(3):103-4, mato/

L_ et alii. Apoio como medida terapêtica no relacionamento enfermeira-paciente. Rev. Esc. Eñe. USP., Săo Paulo, 15(1):43-8, abr. 1981.

et alii. Aceitação, empatia e envolvimento emocional no relacionamento enfermeira-paciente. Rov. Esc. Enf. USP., São Paulo, 16(3):245-53, dez. 1982.

STRONG, P. Relationships in psychiatric nursing. Nurs. Mirror, Sussex, 131(8):35-8, Aug. 21, 1970.

SULLIVAN, H.S. The interpersonal theory of psychiatry. New York, Norton, $1953.393 p$.

TOPALIS, M. \& AGUILERA, D.C. The nurse, the patient and the community. In:

Psychiatric nursing. 7. ed. Saint Louis, C.V. Mosby, 1978 . cap. 6, p.118-51.

TOPF, M. A behavioral checklist for estimating the development of communication skill. J. Nurs. Educ., Thorofore, 8(4) :29-34, Nov. 1969. 
TRAIL, I.D. Wet blishing relationships in psychiatric nursing. New York, Springer, 1966. p.1-6.

TRAVELBEE, J. Intervention in psychiatric nursing: process in tho ono-to-one relationship. Philadelphia, F.A. Davis, 1969. 280p.

TUDOR, G.A. A sociopsychiatric nursing approach to intervention in a problem of mutual withdrawal on a mental hospital ward. Psychiatry, Washington, 15(2):193-217, 1952.

VEnINGA, R. Communication: a patient eye-view. Amer. J. Nurs., New York, 73(2):320-2, Feb. 1973.

VIEIRA, T.P. O processo de comunicasão na enfermagem. Salvador, Centro Editorial e Didático, 1978. 108p.

WALKE, M.A.K. When a patient needs to unburden his feelings. Amer. J. Nurs., New York, 77(7):1164-6, July 1977.

WANDELT, M.A. \& AGER, J.W. Guides and instructions for observations ratings. In: I, p.34-50.

WeBster, M.E. Communicating with dying patients. Nurs. Times, London, 77(23):999-1002, June 4, 1981.

WIEDENBACH, E. The helping art of nursing. Amer. J. Nurs., New York, 63(11):54-7, Nov. 1963.

WLODY, G.S. Effective communication techniques. Nurs. Manage. Chicago, 12(10):19-23, Oct. 1981.

WRIGHT, E.M. \& BURGESS, A.W. Nurse-patient interation. In: BURGESS, A.W. Psychiatric nursing in the hospital and the community. 3. ed. Englewood-Cliffs, Prentice-Hall, 1981. cap. 5 , p.52-74.

YEARWOOD-GRAZETTE, H.S. An anatomy of communication. Nurs. Times, London, 74(41): 1672-9, Oct. 12, 1978.

YURA, H. \& WALSH, M.B. Epilogue. In: —— Human needs and nursing process. New York, Appleton-Century-Crofts, 1978 . cap. 9, p.319-22. 